\title{
Papers of the Postgraduation Program of the Department of Surgery and Anatomy of Ribeirão Preto Medical School, University of São Paulo, Brazil
}

\author{
Orlando de Castro-e-Silva ${ }^{\mathrm{I}}$
}

${ }^{\text {I }}$ Full Professor and Head, Division of Digestive Surgery of the Department of Surgery and Anatomy, Ribeirão Preto Faculty of Medicine, University of São Paulo, Brazil.

Editor in charge of this supplement

By definition, Supplements are collections of papers that deal with related issues or topics, are published as a separate issue of the journal or as part of a regular issue, and are usually funded by sources other than the journal's Publisher. In 2006 we published in Acta Cirúrgica Brasileira a thematic Supplement on liver surgery involving aspects of clinical and experimental research ${ }^{1}$. The papers published in the Supplement were the result of investigations carried out in the Clinical Surgery Postgraduation Program by students and professors of the Division of Digestive Surgery and Liver Transplant. As the central theme, the liver was approached in terms of various aspects contributing to a thematic investigation. In my opinion, this was important from a pedagogic viewpoint, providing the reader with the rare opportunity of evaluating and studying the clinical, surgical, biochemical, physiopathological, and pharmacological aspects of an organ as a central focal point and their relation to liver surgery as a whole and to liver transplantation in particular.

This didactic and pedagogic quality of a Supplement is extremely important because, after peer review, the papers are exposed to the scientific community in a clear and ordered manner based on a pre-established criterion. It is important to emphasize that, in order to be useful for consultation and to fulfill its thematic objective, a Supplement must have the fundamental characteristic of an intimate relation between the papers published in it.

Supplements can serve useful purposes: education, exchange of research information, ease of access to focused content, and improved cooperation between academic and corporate entities. An example is a recent normal issue of the Brazilian Journal of Medical and Biological Research, which emphasized the research tradition of establishing, maintaining and exploiting birth cohort studies of the Ribeirão Preto Medical School ${ }^{2}$. This research line was highlighted as a central theme in this issue of this important Brazilian journal devoted to medical and biological research, which contains a series of twelve papers from the Ribeirão Preto and São Luis birth cohort studies from the Southeast and Northeast of Brazil, respectively. The topics covered in this group of reports vary and include predictors of perinatal health and maternal risk factors, early life determinants of cardiovascular risk factors in childhood and adolescence, use of health services, and a description of dietary characteristics of young adults, amongst other topics. There was also a guide to the background, objectives, sampling and protocols employed across these studies, which, together with similar pieces published in past issues of the same journal, will serve as a very useful starting point, particularly for potential collaborators.

The present Supplement is centered on the clinical and experimental research carried out in the Departament of Surgery and Anatomy of the Ribeirão Preto Medical School, involving papers fully originating from the postgraduation program sensu stricto, developed by both the faculty members and the postgraduate students. Each paper was evaluated by one or two reviewers from the Department of Surgery or from other institutions (peer review). The comments of the reviewers were published together with the respective papers, showing transparency in the evaluation and also providing elements to the readers for a more adequate additional interpretation. The research tradition of the Department of Surgery and Anatomy, the high note attributed to it by CAPES and the high international renown of Acta Cirúrgica Brasileira represent the proper foundation for this Supplement, which has been conceived with the main purpose of providing a proper dissemination of the knowledge produced in an environment devoted to the quality of scientific investigation.

\section{Referênces}

1. Liver Surgery. Experimental and clinical research. Acta Cir Bras. 2006, 21(1).

2. Braz J Med Biol Res, 2007, 40(9). 